\title{
Fluorine F 18 Tetrafluoroborate
}

National Cancer Institute

\section{Source}

National Cancer Institute. Fluorine F18 Tetrafluoroborate. NCI Thesaurus. Code C146693.

A radioconjug ate composed of the sodium-iodide symporter (NIS; SLC5A5) lig and tetrafluoroborate (TFB) labeled with the radionuclide fluorine F 18, with potential use as an imaging agent for NIS-expressing cells upon positron emission tomography (PET). Upon administration, fluorine F 18 tetrafluoroborate targets, binds to, and is taken up by NIS, which is expressed on the surface of cells in the normal thyroid gland, lactating mammary gland and various tumors. Then, NIS-expressing cells can be visualized and NIS expression levels can be assessed using PET. Since iodides are taken up by NIS, this agent may predict the uptake of radioactive iodine by thyroid cancer cells. 